\title{
Focal Arrhythmia Ablation With Multipolar Mapping: Does it Still Make Sense to Stay Off-Grid?
}

\author{
Jay Montgomery ${ }^{1}$ \\ ${ }^{1}$ Vanderbilt University
}

June 22, 2020

\begin{abstract}
Multipolar mapping has primarily been studied in complex arrhythmia substrates or re-entrant circuits. Chieng et al. use a Case-Control design to compare multipolar mapping and point-by-point mapping with an ablation catheter for focal atrial and ventricular tachycardias, showing reduced procedure times and earlier electrograms in the multipolar mapping group but no difference in clinical outcomes. It is plausible that faster mapping and better delineation of earliest signals may translate to improved clinical outcomes if studied in a randomized trial in a larger population. Future multipolar mapping systems will guide the operator toward the focus in real-time and may even triangulate the source in three dimensions, giving an estimate of depth within the myocardium or likely focus in the opposite chamber.
\end{abstract}

Focal Arrhythmia Ablation With Multipolar Mapping: Does it Still Make Sense to Stay Off-Grid?

Jay Montgomery, MD

The history of invasive arrhythmia therapy is paved with technological advances that improve safety and efficiency. Open surgical intervention for accessory pathways was replaced with catheter-based treatments. Direct current fulguration was replaced with radiofrequency ablation. Fluoroscopic imaging was largely supplanted by electroanatomic mapping (EAM). Initially, creation of anatomic maps was performed with the same catheter used for ablation, and this is still sometimes the case. A further refinement of EAM has been the advent of multipolar mapping (MPM) catheters with small electrodes and narrow bipoles which serve the purposes of defining activation patterns and creation of a detailed map without the ability to deliver therapy. These types of catheters give more granular detail of low voltage regions in both atrial and ventricular applications. ${ }^{1,2}$

In most published MPM studies, the MPM catheter was used to define a complex substrate or re-entrant circuit. Multipolar activation mapping of focal arrhythmias is less well-described. In these scenarios, careful delineation of substrate is typically less important, and the area benefitting from detailed activation mapping is often small. MPM is likely helpful in these scenarios, but a more difficult question might be whether it is warranted. For easily-inducible and sustained focal arrhythmias, both point-by-point (PbyP) and MPM should identify the earliest-activating site within a reasonable amount of time, provided the site is accessible and the signal amplitude is large enough to be detected. For more fleeting or unstable arrhythmias, MPM is probably more likely to adequately map the arrhythmia despite a limited total arrhythmia time. Additionally, very low-amplitude signals could potentially be missed by an ablation catheter but detected with MPM. Therefore, it is plausible that a subset of focal arrhythmias would have inadequate mapping unless MPM were used.

In this setting, Chieng et al. used a Case-Control design to compare MPM using the Advisor ${ }^{\mathrm{TM}}$ HD Grid catheter (Abbott Medical, Abbott Park, IL) to PbyP with a contact force-sensing ablation catheter for mapping and ablation of focal atrial and ventricular tachycardias.(CITE THE JCE PAPER HERE) The 
study is limited somewhat by the non-randomized design and relatively low numbers, though the operators were the same and the cases all relatively recent. The primary findings were that acute and medium term outcomes were similar (acute success $85 \%$ vs $81 \%$ MPM vs PbyP; recurrence at 9.4 months $8.7 \%$ MPM vs 9.1\% PbyP, all $\mathrm{p}=\mathrm{NS}$ ) but that mapping and procedure times were shorter in the MPM group (mapping 35 mins vs 53 mins; $p=0.03$; procedure duration 126 mins vs 153 mins in; $p=0.02$ ). Additionally, the earliest recorded electrogram as compared to the $\mathrm{P}$ wave or QRS complex was earlier in the MPM group (39ms pre-QRS) than with the ablation catheter in the PbyP group (33ms pre-QRS, $\mathrm{p}=0.02)$. When compared in the same patients (in only the MPM group), the association seemed to remain (39 ms by MPM catheter vs $35 \mathrm{~ms}$ by ablation catheter), though it was not statistically significant. Despite case-control matching for age and case type (and chamber), overall numbers of atrial and ventricular arrhythmias were not the same in the two groups (more patients with multiple ventricular arrhythmias in the MPM group). Additionally, while a single mapping system was used in the MPM group (Precision, Abbott Medical, Abbott Park, IL), some of the PbyP cohort were mapped with the Carto system (Biosense Webster, Diamond Bar, CA).

It is, of course, not clear how operators decided on multipolar vs PbyP. Given that we are told that the operators were the same, it seems plausible that the patients or arrhythmias were then systematically different (at least to a small degree) if a different approach was used. MPM may have been preferentially chosen in patients with difficult-to-induce or non-sustained arrhythmias or those thought to have a more complex substrate. Given this, the fact that mapping time was 18 minutes less and procedures were 27 minutes shorter in the MPM group seems convincing, as the most obvious confounders would bias toward the opposite. Further, these cases were performed prior to the availability of the LiveView module (Abbott Medical, Abbott Park, IL) which may further truncate mapping times in focal arrhythmias using the HD Grid catheter.

The disparity between the earliest detected electrograms between the two mapping modalities could be due to either failure to detect the same signals despite measuring at the same site or failure of PbyP mapping to find the true earliest sites. The earliest electrograms detected by MPM may have been inherently too low amplitude to be detected by the ablation catheter (with its larger electrode) or could have gone undetected because the wavefront vector was nearly orthogonal to the ablation bipole. Supporting this latter theory, 9 of 27 tachycardias mapped with the HD Grid were found to have a difference of 6 or more milliseconds in the earliest electrogram timing when comparing bipoles along vs across splines. To the extent that wavefront directionality accounts for this disparity in earliest electrograms, the findings from this study utilizing the HD Grid catheter do not necessarily generalize to other MPM catheters without orthogonal bipoles. Further, while this small, non-randomized study showed no difference in clinical outcomes, it is certainly plausible that these earlier-detected signals on the HD Grid catheter may sometimes be anatomically distant enough from the earliest signal seen by an ablation catheter as to reduce the likelihood of arrhythmia recurrence.

It may be tempting to say that a reduction in procedure time translates to a reduction in risk. However, it is within the realm of possibility that some MPM catheter designs could increase risk due to enhanced thrombogenicity or other mechanical factors. ${ }^{3}$ Additionally, some data suggests that exchanging mapping and ablation catheters in a single sheath during ablation of atrial fibrillation increases the risk of silent cerebral microemboli. ${ }^{4}$ Therefore, there is insufficient data to say what, if any, positive or negative safety effect may exist for the use of MPM catheters generally or the HD Grid catheter specifically for focal arrhythmias.

Each currently-available MPM catheter design has likely advantages and disadvantages for various applications. The HD Grid catheter has a relatively fixed form which may not be best-suited for rapidly creating an anatomic shell (such as prior to pulmonary vein isolation) but is unique in its ability to record and integrate information from orthogonal bipoles due to fixed 3-3-3 $\mathrm{mm}$ inter-spline and along-spline spacing. In addition to increasing the likelihood that a bipole is aligned with the wavefront vector, the electrograms from two adjacent and orthogonal bipoles can be used to create a voltage loop assigned to the center of the triangle formed by the two bipoles. The resulting omnipole can be rotated 360 degrees to match the unipolar signal for best fit. The resulting electrogram amplitude is independent of catheter orientation. ${ }^{5-7}$ This novel mapping technique, not yet commercially available in the United States or during the study discussed above, should serve to negate the effects of wavefront directionality on EGM amplitude. 
If these non-randomized results reflect a true effect and MPM with the HD Grid catheter shortens mapping and procedure times but has no effect on success for focal arrhythmias, what should guide practice? Should electrophysiologists weigh the incremental costs of the HD Grid catheter against an additional 27 minutes per procedure? The current, most prudent course may be to carefully consider whether to use MPM for each focal arrhythmia based on the location, ease of inducibility, and anticipated presence or absence of abnormal substrate. However, given the inevitable march of technological progress, MPM will continue to improve, and operators will become accustomed to having it. It seems inevitable that MPM will be increasingly used across most mapping case types as was the case with EAM in general over the past two decades. The mapping system will sense wavefront directionality within a single beat and guide the operator toward the focus in real-time, further decreasing mapping time. It may even be able to triangulate the focus in three dimensions, giving an estimate of the depth within the myocardium or likely focus in the opposite chamber. The real question for the future is: will that same multipolar catheter then be used to deliver therapy, and, if so, what ablation modality will it deliver?

References

1. Anter E, Tschabrunn CM, Josephson ME. High-resolution mapping of scar-related atrial arrhythmias using smaller electrodes with closer interelectrode spacing. Circ Arrhythm Electrophysiol 2015;8:537-45.

2. Tschabrunn CM, Roujol S, Dorman NC, Nezafat R, Josephson ME, Anter E. High-Resolution Mapping of Ventricular Scar: Comparison Between Single and Multielectrode Catheters. Circ Arrhythm Electrophysiol $2016 ; 9$.

3. Nakamura K, Sasaki T, Take Y, et al. Impact of the type of electroanatomic mapping system on the incidence of cerebral embolism after radiofrequency catheter ablation of left atrial tachycardias. Heart Rhythm 2020;17:250-7.

4. Deneke T, Nentwich K, Schmitt R, et al. Exchanging Catheters Over a Single Transseptal Sheath During Left Atrial Ablation is Associated with a Higher Risk for Silent Cerebral Events. Indian Pacing Electrophysiol J 2014;14:240-9.

5. Masse S, Magtibay K, Jackson N, et al. Resolving Myocardial Activation With Novel Omnipolar Electrograms. Circ Arrhythm Electrophysiol 2016;9:e004107.

6. Porta-Sanchez A, Magtibay K, Nayyar S, et al. Omnipolarity applied to equi-spaced electrode array for ventricular tachycardia substrate mapping. Europace 2019;21:813-21.

7. Deno DC, Bhaskaran A, Morgan DJ, et al. High Resolution, Live, Directional Mapping. Heart Rhythm 2020 . 